\title{
Reactive power optimization based on improved bacteria foraging optimization algorithm
}

\author{
Ying $\mathrm{Aa}^{1}$, a Yuanjie $\mathrm{Gao}^{2}$, Hui Sun ${ }^{1}$, Jiwei Zhu and Lizhi Cao ${ }^{1}$ \\ ${ }^{1}$ State grid Wuhan donghu new technology development zone power supply company, Wuhan \\ 430205, China; \\ ${ }^{2}$ State grid Hubei procurement company, Wuhan 430017, China. \\ a504507118@qq.com
}

\begin{abstract}
Keywords: Reactive power optimization, bacteria foraging optimization algorithm, chaos theory, migration operation.
\end{abstract}

\begin{abstract}
Bacteria foraging optimization algorithm have the disadvantages of falling into optimal local optimum easily, and the speed of convergence become to decline obvious in later optimization, this paper established a mathematical model based on minimize the loss of the power network and maintain a good voltage level ,to solve the problem of reactive power optimization for electric power system. Proposed an improved bacteria foraging algorithm, combining the chaotic theory to initialize the basic population of bacteria, so as to improve their global search ability, and adjust the migration operation adaptively, optimizing the efficiency of late convergence, Finally, test on the IEEE -14 node power system, the result improved that the algorithm proposed in this paper has Faster convergence and higher accuracy in the process of the problem of reactive optimization.
\end{abstract}

\section{Introduction}

Power system reactive power optimization refers to the premise of all constraints, when the system topology and operating conditions have given, change the system's current distribution and make some optimal performance indicators, by regulating some control variables [1].Many artificial intelligence-based optimization methods have been developed to solve the reactive power optimization problem in recent years, Mainly include: immune algorithm(IA), particle swarm optimization algorithm(PSO), bacteria foraging optimization algorithm (BFO) [2] etc, achieved some results, but still generally exist the defects of slow convergence or easy to fall into the local optimal. For this reason, this paper proposed an improved bacterial foraging algorithm based on chaos adaptive(CABPO) for power system reactive power optimization, combining chaos in chaos algorithm into bacterial foraging algorithm to improve their global search ability, and adjust the convergence speed and precision by adjust the behavior of bacteria migration behavior. By analyzing the IEEE -14 node and comparing it with particle swarm optimization algorithm , bacteria foraging optimization algorithm, the results show that the CABPO proposed in this paper can solve the reactive power optimization problem more effectively.

\section{Reactive power optimization mathematical model}

\subsection{Objective Function.}

The reactive power optimization mathematical model established by reduce the system active power loss, using the form of penalty function [3]. The target function as follows:

$$
\left\{\begin{array}{l}
\min f=P_{\text {loss }}+\lambda_{V} \sum_{i \in N_{P Q}}\left(\frac{V_{i}-V_{i, \mathrm{lim}}}{V_{i, \max }-V_{i, \mathrm{lim}}}\right)^{2}+\lambda_{Q} \sum_{i \in N_{G}}\left(\frac{Q_{i}-Q_{i, \mathrm{lim}}}{Q_{i, \max }-Q_{i, \mathrm{lim}}}\right)^{2} \\
P_{\text {loss }}=\sum_{i, j \in N}^{N} G_{i j}\left(V_{i}^{2}+V_{j}^{2}-2 V_{i} V_{j} \cos \delta_{i j}\right)
\end{array}\right.
$$


In the type(1): $P_{\text {loss }}$ is the active loss of system; $\lambda_{V}$ and $\lambda_{Q}$ are the failure limit factors of PQ Node voltage and generator power respectively; $G_{i j}$ and $\delta_{i j}$ are the conductance and phase difference between node $i$ and node $j$ respectively.

\subsection{Equality Constraints.}

$$
\begin{cases}P_{i}=V_{i} \sum_{j=1}^{N} V_{j}\left(G_{i j} \cos \delta_{i j}+B_{i j} \sin \delta_{i j}\right) & i \in N_{o} \\ Q_{i}=V_{i} \sum_{j=1}^{N} V_{j}\left(G_{i j} \sin \delta_{i j}-B_{i j} \cos \delta_{i j}\right) & i \in N_{P Q}\end{cases}
$$

In the type (2): $P_{i}$ and $Q_{i}$ are the active power and reactive power respectively; $B_{i j}$ is the Charge value between node $i$ and node $j ; N_{o}$ is the total number of nodes except the balance node.

\subsection{Inequality Constraints.}

\subsubsection{Control Variable Constraints}

$$
\left\{\begin{array}{l}
V_{G i, \text { min }} \leq V_{G i} \leq V_{G i, \max }, i \in N_{G} \\
T_{i, \text { min }} \leq T_{i} \leq T_{i, \max }, i \in N_{T} \\
Q_{c i, \text { min }} \leq Q_{c i} \leq Q_{c i, \max }, i \in N_{C}
\end{array}\right.
$$

\subsubsection{State variable constraint}

$$
\left\{\begin{array}{l}
Q_{G i, \text { min }} \leq V_{G i} \leq V_{G i, \text { max }}, i \in N_{G} \\
V_{d i, \text { min }} \leq V_{d i} \leq V_{d i, \text { max }}, i \in N_{P Q}
\end{array}\right.
$$

In the type(3) and (4): $N_{G}, N_{T}$ and $N_{C}$ are the number of adjustable generators, adjustable transformers and nodes in reactive power compensation device respectively; $V_{G i}, T_{i}$ and $Q_{c i}$ are the terminal voltage of generator, adjustable transformer ratio and compensates capacity of capacitor respectively; $Q_{G i}$ and $V_{d i}$ are generator reactive power output and node voltage of $P Q$.

\section{Application of Chaotic Adaptive Bacterial Foraging Optimization Algorithm in Reactive Power Optimization}

\subsection{Bacterial Foraging Optimization.}

Bacterial foraging optimization (BFO) is a new bionic algorithm to simulate the behavior of Escherichia coli in the human intestine by Toyino in 2002 [4], which mainly includes three steps: chemotaxis, propagation and migration:

Trend. The behavior of bacteria to enrich areas, including two basic movement: tumble and swim. Tumble is looking for a new direction, swimming is to keep the direction of the same movement. The bacteria first selecte one direction to swim randomly, if its fitness value is deteriorated, then tumble and swim in any other direction; otherwise, continue to move in that direction until achieve the maximum number of attempts. The location of bacteria $\mathrm{i}$ changes as type (4) after the $\mathrm{j}$-th chemotaxis operation, k-th copy operation, h-th migration operation:

$$
\theta^{i}(j+1, k, h)=\theta^{i}(j, k, h)+\mathrm{C}(i) \phi(j)
$$

In the type(5): $C(i)>0$ refers the unit step of swing; $\phi(j)$ refers the forward direction which select randomly after tumble operation.

Breeding. The breeding process of bacteria follows the natural criteria of "survival of the fittest". Expressed as sort the bacterial energy by size after a round of chemotaxis, eliminate the half inferiority of the individual, copy the remaining half of the elite individual to maintain the bacterial population size.

Migrate. The area of bacteriological life may occur drastic changes at any time, which can cause bacteria to die or migrate to new areas. Specific performance as the bacteria death in a certain probability $P_{e d}$, and generate a new individual instead randomly, increasing the possibility of jumping out of the local optimal solution. The fitness of bacteria i changes as type (5) after one chemotaxis. 


$$
\left\{\begin{array}{l}
J_{c c}(\theta, P(j, k, h))=\sum_{i=1}^{S} J_{c c}\left(\theta, \theta^{i}(j, k, h)\right)=\sum_{i=1}^{S}\left[-d_{a} \exp \left(-w_{a} \sum_{m=1}^{D}\left(\theta_{m}-\theta_{m}^{i}\right)^{2}\right)\right]+\sum_{i=1}^{S}\left[h_{r} \exp \left(-w_{r} \sum_{m=1}^{D}\left(\theta_{m}-\theta_{m}^{i}\right)^{2}\right)\right] \\
J(i, j+1, k, h)=J(i, j, k, h)+J_{c c}\left(\theta^{i}(j+1, k, h), P(j+1, k, h)\right)
\end{array}\right.
$$

In the type(6): $J_{c c}$ is the effect of signal transmission between bacteria; ${ }_{a}, w_{a}, h_{r}, w_{r}$ are the depth of gravity, width of the gravitational force, height of the repulsive force, and the width of the repulsive force respectively.

\subsection{Chaotic Adaptive Bacterial Foraging Optimization Algorithm. \\ 3.2.1 Chaos Initialization Bacteria Population}

In basic BFO, the initial bacterial population is generated randomly, resulting in an uneven distribution in the spatial distribution, and affects the global search ability of the algorithm. To overcome this defect, this paper proposes to initialize the bacterial population by using the logistic mapping, optimize the individual quality, and improve its global search ability through the use of chaotic ergodicity. The mathematical expression of logistic mapping is:

$$
y_{k+1}=\mu y_{k}\left(1-y_{k}\right), k=0,1,2 \ldots
$$

In the type(7): $k$ is the number of iterations; $\mu$ is the parameter that controls the chaotic behavior of the system; $y_{k}$ is a chaotic variable; The system will fall into chaotic state, and traversals in 0 to 1, When The $\mu$ Values 4.

\subsubsection{Adaptive Migration Operation}

The migration operation re-distribution the bacterial groups only in a fixed probability in basic BFO, which may lead to "escape" phenomenon easily, That is, make the bacteria to migrate away for the population which has been found the optimal location, loss the elite individuals, reducing the convergence efficiency of the algorithm. So this paper proposes to adjust the migration probability adaptively, look the fitness of the bacteria as evaluation carrier, when the energy value of bacteria $i$ is less than the minimum energy value in the population, then consider that this bacteria is far from the optimal location, and its migration probability becomes larger adaptively, otherwise, the migration probability becomes smaller adaptively. All bacteria change their migration probability follow the formula (7):

$$
P_{\text {self }}(i)=\frac{J_{\max }-J_{i}}{J_{\max }-J_{\min }} \cdot P_{e d}
$$

In the type(8): $J_{i}$ is the fitness of bacteria i; $J_{\max }$ and $J_{\min }$ are the maximum and minimum energy values in the current bacterial population respectively.

\subsection{Basic Optimization Steps of the Improved Algorithm}

1) Initialize the bacteria population scale $S$, the chemotactic number $N_{c}$, the replication number $N_{e d}$, basic migration probability $P_{\text {ed }}$, swim number $N_{s}$, etc.

2) Initialize the bacterial population within the variable design range by tent mapping

3) Chemotaxis: Update the position of each bacterium by flip, if the position is better, move forward.

4Replication operation: sort the bacteria according to their accumulation fitness, out of the poor value of half of the bacteria, Self-splitting of the remaining bacteria.

5) Migration operation: adjust the migration probability of bacteria in accordance with the formula adaptively, out of the bacteria when its migration probability is greater than the given probability.

6) Judge whether it meet the termination conditions. If the maximum number of iterations, putout the optimal solution; otherwise, return to step (3) and continue the iterative calculation.

\section{Simulation Analysis}

Test on IEEE 14-node system for reactive power optimization, and compare CABPO with PSO and BPO. The settings of the parameters in the algorithms are as follows: bacteria population size is 35 , chemotaxis number is 5 , replication number is 4 , migration number is 2 , basic migration 
probability is 0.25 , and swimming number is 3 . In IEEE 14 -node system, the standard capacity is 100MVA, the scope of generator terminal voltage is $[0.9,1.1]$, the variable ratio range of the adjustable transformer is $[0.9,1.1]$, divided into four points,the adjusting step length is 0.025 , the upper limit of reactive power compensation capacity is $0.5 \mathrm{p} . \mathrm{u}$, divided into ten points, the adjusting step length is 0.05 . Operating the three algorithms independently for 50 times, the average optimization results are obtained as it shown in table 1.

Table 1. Average optimization results of IEEE 14bus system

\begin{tabular}{cc}
\hline Algorithm & Network Loss/p.u \\
\hline PSO & 0.1365311 \\
BPO & 0.1362024 \\
CABPO & 0.1359719 \\
\hline
\end{tabular}

As it can be seen from Table 1: CABPO required the minimum of network loss, range from the initial 0.15872 p.u down to 0.13597 p.u, loss rate about $14.33 \%$, means that helps improve the system power factor. And the optimization effect is better than the other two algorithms.

Figure 1 shows the average convergence curve of the active loss of the three algorithms in solving the reactive power optimization problem. Among them, CABFO declined quicker in initial iteration is due to introduced the thought of chaos; At the same time, the migration operation is adjusted adaptively, which makes the algorithm have strong global search ability, stronger stability in the later stage of the iteration, and easier to jump out of local optimum.

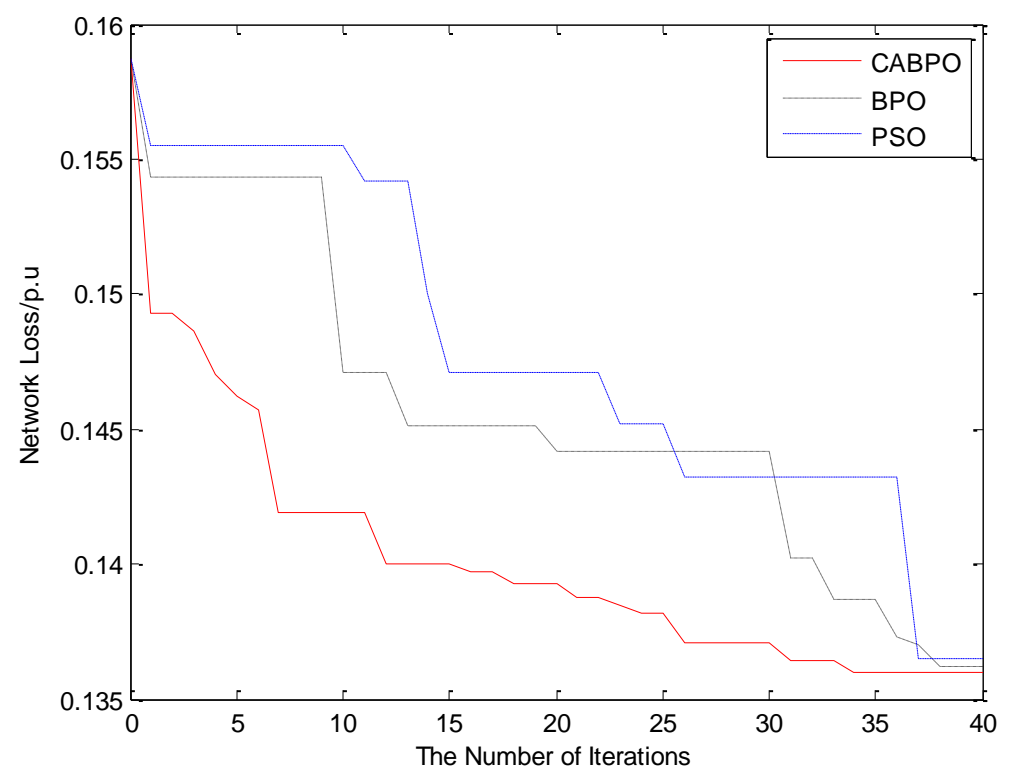

Fig.1 Optimization values curve of three algorithms

\section{Summary}

This paper proposed an improved CABFO algorithm for reactive power optimization of power system. The initialization and migration operations are optimized in basic BFO, using Logistic mapping to initialize the bacterial population, which improved the ergodicity of the initial solution and its global search ability; while adjusting the migration probability of each bacterium adaptively, which improved the accuracy of the algorithm. The simulation results of IEEE-14 nodes show that the CABFO algorithm proposed in this paper is better than PSO and BPO algorithm in dealing with reactive power optimization. 


\section{References}

[1]. He R, Taylor G A, Song Y H. Multi-objective optimal reactive power flow including voltage security and demand profile classification [J]. International Journal of Electrical Power \& Energy Systems, 2008, 30(5):327-336.

[2]. Li Zhihuan, Duan Xianzhong. Comparison and Analysis of Multi-objective Evolutionary Algorithm for Reactive Power Optimization [J]. Proceedings of the CSEE, 2010, 30(10): 57-65(in Chinese).

[3]. Kenedy J, Eberhart R. Particle swarm optimization[C]. Proceedings of IEEE International Conference on Neural Networks, Perth, Australia, 1995(4):1942-1984.

[4]. A Abraham, et al. Analysis of reproduction operator in bacterial foraging optimization algorithm [C]. Proceedings of 2008 IEEE Conference on Evolutionary Computation, New York: IEEE, 2008:1476-1493. 